\title{
Implications of low energy supersymmetry breaking at the Fermilab Tevatron
}

\author{
Savas Dimopoulos \\ Theoretical Physics Division, CERN, CH-1211 Geneva 23, Switzerland, \\ and Physics Department, Stanford University, Stanford, California 94305 \\ Scott Thomas and James D. Wells \\ Stanford Linear Accelerator Center, Stanford, California 94309 \\ (Received 7 May 1996)
}

\begin{abstract}
The signatures for low energy supersymmetry breaking at the Fermilab Tevatron are investigated. It is natural that the lightest standard model superpartner is an electroweak neutralino, which decays to an essentially massless Goldstino and photon, possibly within the detector. In the simplest model of gauge-mediated supersymmetry breaking, the production of right-handed sleptons, neutralinos, and charginos leads to a pair of hard photons accompanied by leptons and/or jets with missing transverse energy. The relatively hard leptons and softer photons of the single $e^{+} e^{-} \gamma \gamma+\mathbb{E}_{T}$ event observed by CDF implies this event is best interpreted as arising from left-handed slepton pair production. In this case the rates for $l^{ \pm} \gamma \gamma+\mathbb{E}_{T}$ and $\gamma \gamma+\boldsymbol{E}_{T}$ are comparable to that for $l^{+} l^{-} \gamma \gamma+\boldsymbol{E}_{T}$. [S0556-2821(96)04717-0]

PACS number(s): 14.80.Ly, 11.30.Qc, 12.60.Jv
\end{abstract}

\section{INTRODUCTION}

If supersymmetry at the electroweak scale is established, one of the important questions to be addressed experimentally is the scale and mechanism of supersymmetry breaking. It is often assumed that supersymmetry is broken in a hidden sector at a very high scale, with the breaking transmitted to the visible sector by gravitational strength interactions. It is possible, however, that supersymmetry is broken at a scale not too far above the electroweak scale, with the breaking transmitted by nongravitational interactions $[1,2]$. In this case the gravitino is naturally the lightest supersymmetric particle. The longitudinal component of the gravitino, the Goldstone fermion of supersymmetry breaking, or Goldstino $G$, couples to ordinary matter through interactions suppressed only by the supersymmetry-breaking scale [3]. This allows the lightest standard model supersymmetric particle to decay to its partner plus the Goldstino. In the simplest models the lightest standard model superpartner is a neutralino $\chi_{1}^{0}$; the dominant decay mode over much of the parameter space is $\chi_{1}^{0} \rightarrow \gamma G$ [3-6]. For a supersymmetry-breaking scale below a few thousand $\mathrm{TeV}$ this decay can take place inside the detector. Within the context of the usual supersymmetric standard model, with high scale supersymmetry breaking, radiative decays of neutralinos are not generic, but can be achieved by tuning parameters $[7,8]$. The presence of two hard photons and missing transverse energy in the final state is therefore a distinctive and generic signature for low scale supersymmetry breaking.

At a hadron collider the production rates for supersymmetric states and subsequent cascade decays are determined by both the masses and gauge couplings. The form of the superpartner mass spectrum is determined by the interactions which transmit supersymmetry breaking to the visible sector. With low scale supersymmetry breaking, one of the simplest possibilities is that these interactions are just the ordinary gauge interactions $[1,2,4]$. The superpartner masses are then roughly proportional to their gauge couplings squared. This generally implies that the gluino and squarks are too heavy to be produced at the Fermilab Tevatron. The largest production rates are for sleptons, charginos, and neutralinos. As discussed below, the relative rates and kinematics in the various channels can be sensitive to the superpartner mass spectrum and, in turn, to details of the messenger sector in which supersymmetry is broken.

To illustrate the sensitivity of different channels to the form of the messenger sector, we consider a number of scenarios which can arise with gauge-mediated supersymmetry breaking and identify important generic features of the signatures. In the next section the minimal model of gauge mediation is reviewed. In this model, if $\chi_{1}^{0}$ is mostly gaugino, its production is suppressed by the large squark masses. Pair production of right-handed sleptons, and subsequent cascade decays through $\chi_{1}^{0}$, leads to the final state $l^{+} l^{-} \gamma \gamma+\mathbb{E}_{T}[5,6]$. In addition, chargino and neutralino pair production leads to the final states $W W \gamma \gamma+\mathbb{E}_{T}$ and $W l^{+} l^{-} \gamma \gamma+\boldsymbol{E}_{T}$ or $W Z \gamma \gamma+\boldsymbol{E}_{T}$. In Sec. III the minimal model with an approximate $\mathrm{U}(1)_{R}$ symmetry is considered. In this case, the gauginos are lighter than in the minimal model, leading to relatively larger production rates for charginos and neutralinos. In Sec. IV the minimal model is considered in the case in which $\chi_{1}^{0}$ is roughly equal mixtures of gaugino and Higgsino. This gives rise to additional final states including $j j \gamma \gamma+\boldsymbol{E}_{T}$ and $l^{ \pm} \gamma \gamma+\boldsymbol{E}_{T}$. In Sec. V models in which lefthanded sleptons are lighter than in the minimal model are considered. Pair production of left-handed sleptons gives, in addition to $l^{+} l^{-} \gamma \gamma+\boldsymbol{E}_{T}$, the final states $l^{ \pm} \gamma \gamma+\boldsymbol{E}_{T}$ and $\gamma \gamma+\boldsymbol{E}_{T}$ at comparable rates. For definiteness we assume throughout that the lightest standard model superpartner is a neutralino and that its decay to a photon plus Goldstino is prompt. Consequences of relaxing the latter assumption are discussed in the final section.

A single event of the type $e^{+} e^{-} \gamma \gamma+\boldsymbol{E}_{T}$ has been reported by the Collider Detector at Fermilab (CDF) Collaboration [9]. Such a signature is consistent with slepton pair 
production and low scale supersymmetry breaking [6,8]. In Sec. VI we consider this interpretation of the event within the context of the models discussed below. The kinematics of the event, namely, hard leptons and somewhat softer photons, and apparent lack of many other events with jets in the final state, is most easily accommodated with left-handed slepton production. In this case, the additional final states mentioned above should be seen at comparable rates.

\section{MINIMAL MODEL OF GAUGE-MEDIATED SUPERSYMMETRY BREAKING}

If supersymmetry is broken at a low scale, the ordinary gauge interactions can act as messengers of supersymmetry breaking. The simplest possible messenger sector, which preserves the successful prediction of $\sin ^{2} \theta_{W}$ at low energy, is fields which possess the quantum numbers of a single $\mathbf{5}+\overline{\mathbf{5}}$ of SU(5). The triplets $q$ and $\bar{q}$ and doublets $\ell$ and $\bar{l}$, of $\mathbf{5}+\overline{\mathbf{5}}$, couple to a single background field $S$ through a superpotential $W=S\left(\lambda_{1} q \bar{q}+\lambda_{2} \ell \bar{\ell}\right)$. The field $S$ breaks both $\mathrm{U}(1)_{R}$ and supersymmetry through its scalar and auxiliary components, respectively. Integrating out the messenger sector fields gives rise radiatively to both scalar and gaugino masses. The visible sector gluino and squarks in this model are heavy enough to be beyond the reach of the Tevatron. The masses of the left-handed sleptons, $W$-inos [partners of the $\mathrm{SU}(2)_{L}$ gauge bosons], right-handed sleptons, and $B$-ino [partner of the $\mathrm{U}(1)_{Y}$ gauge boson], are in the ratio $2.5: 2: 1.1: 1$. We will refer to this model as the minimal gauge-mediated (MGM) model of supersymmetry breaking. The dimensionful terms in the Higgs sector required to break the $\mathrm{U}(1)_{\mathrm{PQ}}$ and $\mathrm{U}(1)_{R-\mathrm{PQ}}$ symmetries, $W=\mu H_{1} H_{2}$ and $V=-m_{12}^{2} H_{1} H_{2}+$ H.c., must arise from additional interactions $[4,10,11]$ and may be taken as free parameters in the minimal model. Values of $|\mu|$ larger than roughly $150 \mathrm{GeV}$ are mildly preferred in order to suppress charged Higgs contributions to $B(b \rightarrow s \gamma)$ [11]. For the mass ranges considered below, the lightest two neutralinos $\chi_{1}^{0}$ and $\chi_{2}^{0}$ and lightest chargino $\chi_{1}^{ \pm}$are then mostly gaugino, with small Higgsino mixtures. In the $\mu \gg m_{\chi_{1}^{0}}$ limit, the spectrum of light states is in the ratios given above, and the most important parameter which determines the phenomenology at the Tevatron is just the overall scale.

The production rate for the light states depends on both the masses and charges. If the lightest neutralinos are mostly gaugino, $\chi_{1}^{0}$ is mostly $B$-ino. Pair production of $\chi_{1}^{0} \chi_{1}^{0}$ through off-shell $Z^{*}$ exchange is then suppressed by a small coupling, and through $t$ - and $u$-channel squark exchange by the large squark masses. However, pair production of $\widetilde{l}_{R} \widetilde{l}_{R}$ through off-shell $\gamma^{*}$ and $Z^{*}$, and subsequent cascade decay $\widetilde{l}_{R} \rightarrow l \chi_{1}^{0}$, leads to the final state $l^{+} l^{-} \gamma \gamma+\boldsymbol{E}_{T}[5,6]$. In addition, pair production of charginos and neutralinos through an off-shell $W^{*}$ (via coupling to the $W$-ino components) leads to comparable production rates for $\chi_{1}^{+} \chi_{1}^{-}$and $\chi_{2}^{0} \chi_{1}^{ \pm}$. For large $|\mu|$ the neutralino $\chi_{2}^{0}$ decays predominantly by $\chi_{2}^{0} \rightarrow \widetilde{l}_{R} l$. For any reasonable $\mu$ and $m_{\chi_{1}^{ \pm}}>m_{\chi_{1}^{0}}+m_{W}$, the chargino $\chi_{1}^{ \pm}$decays predominantly through its Higgsino components to the Higgsino components of $\chi_{1}^{0}$ by $\chi_{1}^{ \pm} \rightarrow \chi_{1}^{0} W$. On the other hand, for $m_{\chi_{1}^{ \pm}}<m_{\chi_{1}^{0}}+m_{W}, \chi_{1}^{ \pm}$de-
TABLE I. Production cross sections $(\mathrm{fb})$ for each lepton flavor within the MGM for $m_{\chi_{1}^{0}}=100 \mathrm{GeV}, \mu \gg m_{\chi_{1}^{0}}$, and $m_{\tilde{l}_{R}}=110 \mathrm{GeV}$, as discussed in Sec. II. The center of mass energy is $1.8 \mathrm{TeV}$. Each final state has $\mathbb{E}_{T}$. The total cross sections in each channel are summed over all lepton flavors.

\begin{tabular}{cccc}
\hline \hline & $l^{+} l^{-} \gamma \gamma$ & $W l^{+} l^{-} \gamma \gamma$ & $W W \gamma \gamma$ \\
\hline$\widetilde{l_{R}} \widetilde{l}_{R}$ & 6 & - & - \\
$\chi_{2}^{0} \chi_{1}^{ \pm}$ & - & 11.5 & - \\
$\chi_{1}^{+} \chi_{1}^{-}$ & - & - & 18.8 \\
\hline Total & 18 & 34.4 & 18.8 \\
\hline \hline
\end{tabular}

cays to three-body final states predominantly through offshell $W^{*}$ and $\widetilde{l_{R}^{*}}$ The total cross sections which arise at the Tevatron in this model with $m_{\chi_{1}^{0}}=100 \mathrm{GeV}$ and $\mu \gg m_{\chi_{1}^{0}}$ are given in Table I. In this case $\chi_{1}^{0}$ is pure $B$-ino and $\chi_{2}^{0}$ and $\chi_{1}^{ \pm}$are pure $W$-ino. In the $\sin ^{2} \theta_{W} \rightarrow 0$ limit $\sigma\left(\chi_{2}^{0} \chi_{1}^{ \pm}\right)=2 \sigma\left(\chi_{1}^{+} \chi_{1}^{-}\right)$.

For finite $\mu$ constructive or destructive interference with the Higgsino mixtures in $\chi_{2}^{0}$ and $\chi_{1}^{ \pm}$can significantly affect the cross sections. For example, with $\mu=-250 \mathrm{GeV}$, $m_{B}=100 \mathrm{GeV}$, and $\tan \beta \equiv\left\langle H_{2}\right\rangle /\left\langle H_{1}\right\rangle=2, \sigma\left(\chi_{2}^{0} \chi_{1}^{ \pm}\right) \simeq 25.4$ $\mathrm{fb}$ and $\sigma\left(\chi_{1}^{+} \chi_{1}^{-}\right) \simeq 13.7 \mathrm{fb}$. The branching ratios can also be modified for finite $\mu$. For the above parameters, $m_{\chi_{2}^{0}}-m_{\chi_{1}^{0}}>m_{Z}$ and so $\chi_{2}^{0}$ decays predominantly through its Higgsino components to the Higgsino components of $\chi_{1}^{0}$ by $\chi_{2}^{0} \rightarrow \chi_{1}^{0} Z$. The final states $W l^{+} l^{-} \gamma \gamma+\boldsymbol{E}_{T}$ are then replaced by $W Z \gamma \gamma+\boldsymbol{E}_{T}$.

The total rates of course depend on the overall scale, but the relative rates in the various channels are a slow function of the overall scale. The final states $l^{+} l^{-} \gamma \gamma+\boldsymbol{E}_{T}$, $W l^{+} l^{-} \gamma \gamma+\boldsymbol{E}_{T}$, and $W W \gamma \gamma+\boldsymbol{E}_{T}$, therefore, represent an important test of the MGM model in the large $|\mu|$ limit. The relative rates in the $W l^{+} l^{-} \gamma \gamma+\boldsymbol{E}_{T}$ and $W Z \gamma \gamma+\mathbb{E}_{T}$ are sensitive to the magnitude of $\mu$, as discussed above. In addition, if the usual gauge interactions are the dominant messengers of supersymmetry breaking, it follows that the right-handed sleptons are essentially degenerate. Final states for each lepton flavor should have equal rates. Because of the relatively large mass of the left-handed sleptons, pair production of $\widetilde{l}_{L} \widetilde{l}_{L}$ through off-shell $\gamma^{*}$ and $Z^{*}$, and $\widetilde{\nu}_{L} \widetilde{l}_{L}$ through offshell $W^{*}$, is suppressed in the MGM model. For example, with the parameters given in Table I, $\sigma\left(\widetilde{\nu}_{L} \widetilde{l}_{L}\right) / \sigma\left(\widetilde{l_{R}} \widetilde{l}_{R}\right)$ $\simeq 0.04$ and $\sigma\left(\widetilde{l_{L}} \widetilde{l_{L}}\right) / \sigma\left(\widetilde{l_{R}} \widetilde{l_{R}}\right) \simeq 0.025$.

An important feature of the MGM model is the kinematics of the partons in the final states. Since the mass splitting between $\widetilde{l}_{R}$ and the $B$-ino is so small, the decay $\widetilde{l}_{R} \rightarrow l \chi_{1}^{0}$ results in fairly soft leptons. In contrast, for the decay $\chi_{1}^{0} \rightarrow \gamma G$, the photon receives half the $\chi_{1}^{0}$ mass in the rest frame, resulting in a larger average photon energy. In addition, since $\chi_{1}^{0}$ is generally boosted in the laboratory frame, the photon $E_{T}$ spectrum is much flatter than that of the leptons. The $E_{T}$ and $\boldsymbol{E}_{T}$ for the $l^{+} l^{-} \gamma \gamma+\boldsymbol{E}_{T}$ final state with the parameters of Table I are shown in Fig. 1 [12]. This illustrates how the kinematics can be used to infer mass splittings within a decay chain. 


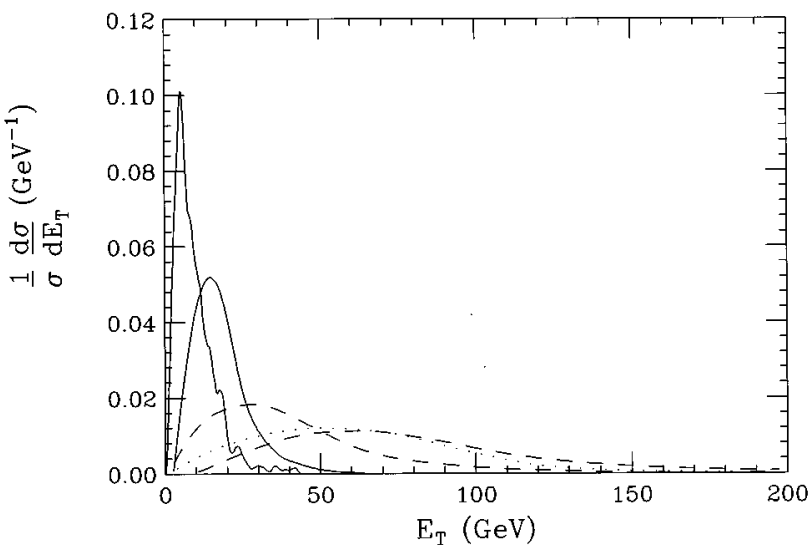

FIG. 1. The $E_{T}$ and $\mathbb{E}_{T}$ spectra for the $l^{+} l^{-} \gamma \gamma+\mathbb{E}_{T}$ channel in the MGM model with the parameters given in Table I. The two solid lines are the $E_{T}$ distributions of the hard and soft electrons. Similarly, the dashed lines are the $E_{T}$ distributions of the hard and soft photons. The dotted line is the $\mathbb{E}_{T}$ distribution.

\section{MINIMAL GAUGE MEDIATION WITH AN APPROXIMATE U(1) $R$ SYMMETRY}

Scalar masses require supersymmetry breaking, whereas gaugino masses require the breaking of both supersymmetry and $\mathrm{U}(1)_{R}$ symmetry. In the MGM model a single field $S$ is assumed to communicate the breaking of both $\mathrm{U}(1)_{R}$ and supersymmetry to the messenger sector. This is the origin of the relation between the gaugino and scalar masses. In general, however, these two symmetries can be broken in different sectors. As a simple example, consider a messenger sector with fields which carry the quantum numbers of two generations of $\mathbf{5}+\overline{\mathbf{5}}$, with superpotential $W=\lambda X\left(\mathbf{5}_{1} \overline{\mathbf{5}}_{1}\right.$ $\left.+\xi^{2}\right)+\lambda^{\prime} S\left(\mathbf{5}_{1} \overline{\mathbf{5}}_{2}+\mathbf{5}_{2} \overline{\mathbf{5}}_{1}\right)$. For $\lambda^{\prime} S>\xi, \mathbf{5}_{i}=\overline{\mathbf{5}}_{i}=0$, and $X$ and $S$ are undetermined at the tree level. Supersymmetry is broken for $\xi \neq 0$, while a $\mathrm{U}(1)_{R}$ symmetry is broken for $\lambda X \neq 0$. For $\lambda X \ll \lambda^{\prime} S$ there is an approximate $\mathrm{U}(1)_{R}$ symmetry, and the visible sector gauginos can be significantly lighter than the scalars.

It is possible then that the small mass splitting between $\tau_{R}$ and the $B$-ino which exists in the MGM model is larger in more general models. This has the effect of decreasing the $\widetilde{l}_{R} \widetilde{l}_{R}$ production rate relative to the $\chi_{1}^{+} \chi_{1}^{-}$and $\chi_{2}^{0} \chi_{1}^{ \pm}$ rates. For large enough mass splitting, it is possible that $m_{\tau_{R}}>m_{\chi_{2}^{0}}-m_{\chi_{1}^{0}}$. The neutralino $\chi_{2}^{0}$ then decays predominantly through its Higgsino components to the Higgsino components of $\chi_{1}^{0}$ by $\chi_{2}^{0} \rightarrow \chi_{1}^{0} Z$. The relative rates in the final states $l^{+} l^{-} \gamma \gamma+\mathbb{E}_{T}, W l^{+} l^{-} \gamma \gamma+\mathbb{E}_{T}$, and $W Z \gamma \gamma+\boldsymbol{E}_{T}$ are therefore sensitive to the mass splitting between $\widetilde{l}_{R}$ and the $B$-ino in the MGM model with an approximate $\mathrm{U}(1)_{R}$ symmetry. Independent of the $l^{+} l^{-} \gamma \gamma+\boldsymbol{E}_{T}$ final state, if $m_{\tau_{L}}$, $m_{\widetilde{\nu}_{L}}>m_{\chi_{2}^{0}}, W W \gamma \gamma+\boldsymbol{E}_{T}$ and the sum of $W l^{+} l^{-} \gamma \gamma+\boldsymbol{E}_{T}$ and $W Z \gamma \gamma+\boldsymbol{E}_{T}$ final states represent an important test of whether the two lightest neutralinos are mostly gaugino within low-scale gauge-mediated supersymmetry breaking.

An important distinction for models with an approximate $\mathrm{U}(1)_{R}$ symmetry is the kinematics of the partons in the $l^{+} l^{-} \gamma \gamma+\mathbb{E}_{T}$ final states. Since the mass splitting between $\widetilde{l}_{R}$ and the $B$-ino can be larger than in the MGM model, the decay $\widetilde{l_{R}} \rightarrow l \chi_{1}^{0}$ gives rise to harder leptons. Even with a relatively small number of events it should be possible to distinguish between models with the MGM mass relations and more general models with larger mass splittings.

\section{MINIMAL GAUGE MEDIATION WITH HIGGSINO PRODUCTION}

The four neutralinos of the minimal supersymmetry standard model are in general a mixture of gauginos and Higgsinos. For $\mu$ comparable to the gaugino masses, pair production of neutralino and charginos through the Higgsino components can give rise to additional important channels. An example which illustrates the case in which the lightest neutralinos are roughly equal mixtures of gauginos and Higgsinos is for the parameters $\mu=-160 \mathrm{GeV}, m_{B}=150$ $\mathrm{GeV}$, and $\tan \beta=2$. This choice of $\mu$ represents a low value which is still marginally consistent with $B(b \rightarrow s \gamma)$ [11]. The neutralino mass eigenvalues are then $144,169,177$, and $322 \mathrm{GeV}$. The two lightest neutralinos $\chi_{1}^{0}$ and $\chi_{2}^{0}$ are roughly equal mixtures of $B$-inos and the symmetric combination of Higgsinos. The neutralino $\chi_{3}^{0}$ is mostly the antisymmetric combination of Higgsinos, while $\chi_{4}^{0}$ is mostly $W$-ino. The coupling of an off-shell $Z^{*}$ to pairs of nearly symmetric or antisymmetric Higgsinos is suppressed, but the coupling of a $Z^{*}$ to a symmetric Higgsino and antisymmetric Higgsino is unsuppressed. The dominant neutralino pair production with the above parameters is therefore for $\chi_{1}^{0} \chi_{3}^{0}$. Since

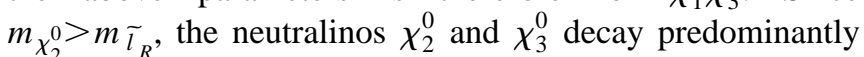
by $\chi_{i}^{0} \rightarrow \widetilde{l}_{R} l$. Production of $\chi_{1}^{0} \chi_{3}^{0}$ therefore gives the final state $l^{+} l^{-} \gamma \gamma+\boldsymbol{E}_{T}$. In more general models with $m_{\tilde{l}_{R}}>m_{\chi_{2}^{0}}$ the neutralinos decay predominantly to threebody final states through off-shell $\widetilde{l_{R}^{*}}$ and $Z^{*}$.

The chargino $\chi_{1}^{ \pm}$is mostly Higgsino, while $\chi_{2}^{ \pm}$is mostly $W$-ino, with masses 168 and $322 \mathrm{GeV}$, respectively. The chargino $\chi_{1}^{ \pm}$decays predominantly to three-body final states through an off-shell $W^{*}$. Pair production of $\chi_{1}^{+} \chi_{1}^{-}$through off-shell $\gamma^{*}$ and $Z^{*}$ then leads to $X \gamma \gamma+\mathbb{E}_{T}$ final states, where $X=l^{+} l^{-}, l^{ \pm} j j$, and $4 j$. In addition, production of $\chi_{1}^{ \pm} \chi_{i}^{0} \quad i=1,2,3$, through an off-shell $W^{*}$, leads to the final states $X \gamma \gamma+\boldsymbol{E}_{T}$, where $X=l^{ \pm}, l^{+} l^{-} l^{\prime \pm}, l^{+} l^{-} j j$, and $j j$. The production cross sections for this set of parameters are summarized in Table II. The mostly $W$-ino states $\chi_{4}^{0}$ and $\chi_{2}^{ \pm}$are too heavy to have appreciable rates with these parameters.

One feature from Table II which generically distinguishes production through Higgsino components is the relatively large rate for $\chi_{1}^{ \pm} \chi_{1}^{0}$. This leads to the final states $j j \gamma \gamma+\mathbb{E}_{T}$ and $l^{ \pm} \gamma \gamma+\boldsymbol{E}_{T}$, which do not occur in the mostly gaugino scenario. As another example, for the parameters given in Table I with $\mu=250 \mathrm{GeV}$, the Higgsino components of $\chi_{1}^{0}$ and $\chi_{1}^{ \pm}$give $\sigma\left(\chi_{1}^{0} \chi_{1}^{ \pm}\right) \simeq 40 \mathrm{fb}$. In addition, the other cross sections are increased to $\sigma\left(\chi_{2}^{0} \chi_{1}^{ \pm}\right) \simeq 105 \mathrm{fb}$, and $\sigma\left(\chi_{1}^{+} \chi_{1}^{-}\right) \simeq 54 \mathrm{fb}$.

An additional feature for production of neutralinos and charginos with large Higgsino fractions is the kinematics of the final states. Since Higgsino masses are determined by one mass parameter $(\mu)$, they tend to be fairly degenerate, whereas the gauginos are more split $\left(M_{2} \simeq 2 M_{1}\right)$. For 
TABLE II. Production cross sections (fb) for each lepton flavor within the MGM for $\mu=-160 \mathrm{GeV}$, $m_{\tilde{B}}=150 \mathrm{GeV}$, and $m_{\widetilde{e}_{R}}=165 \mathrm{GeV}$, as discussed in Sec. IV. The center of mass energy is $1.8 \mathrm{TeV}$. Each final state has $\boldsymbol{E}_{T}$. The total cross sections in each channel are summed over all lepton flavors.

\begin{tabular}{|c|c|c|c|c|c|c|c|c|}
\hline & $l^{ \pm} \gamma \gamma$ & $l^{+} l^{-} \gamma \gamma$ & $l^{+} l^{-} l^{ \pm} \gamma \gamma$ & $l^{+} l^{-} l^{\prime \pm} \gamma \gamma$ & $l^{ \pm} j j \gamma \gamma$ & $l^{+} l^{-} j j \gamma \gamma$ & $j j \gamma \gamma$ & $4 j \gamma \gamma$ \\
\hline$\widetilde{l}_{R} \widetilde{l}_{R}$ & - & 1.0 & - & - & - & - & - & - \\
\hline$\chi_{1}^{0} \chi_{3}^{0}$ & - & 4.3 & - & - & - & - & - & - \\
\hline$\chi_{1}^{+} \chi_{1}^{-}$ & 一 & - & - & - & 1.9 & - & - & 5.7 \\
\hline$\chi_{1}^{ \pm} \chi_{1}^{0}$ & 2.3 & - & - & - & - & - & 14.0 & - \\
\hline$\chi_{1}^{ \pm} \chi_{2}^{0}$ & - & - & 0.5 & 0.5 & - & 3.0 & - & - \\
\hline$\chi_{1}^{ \pm} \chi_{3}^{0}$ & 一 & 一 & 0.9 & 0.9 & 一 & 5.1 & - & - \\
\hline Total & 6.9 & 15.9 & 4.2 & 8.4 & 5.7 & 24.3 & 14.0 & 5.7 \\
\hline
\end{tabular}

$\mu \sim M_{1}$ this leads to mass splittings among the light neutralinos which are much smaller than the overall scale. Thus, the leptonic and/or hadronic activity coming from cascade decays down to $\chi_{1}^{0}$ tends to be much softer than the photons arising from $\chi_{1}^{0} \rightarrow \gamma G$.

\section{NONMINIMAL MODELS WITH LEFT-HANDED SLEPTON PRODUCTION}

The ratio $m_{\tilde{l}_{L}} / m_{\tilde{l}_{R}} \simeq 2.3$ is fixed in the MGM model by the form of the messenger sector and the relative magnitude of the $\mathrm{SU}(2)_{L}$ and $\mathrm{U}(1)_{Y}$ gauge couplings. In more general models this ratio can be modified. For example, additional gauge interactions under which $\widetilde{l}_{R}$ is charged, or direct Yukawa couplings of the messengers with the Higgs or matter multiplets, can in general reduce this ratio. Pair production of $\widetilde{l}_{L} \widetilde{l}_{L}, \widetilde{l_{L}} \widetilde{\nu}_{L}$, and $\widetilde{\nu}_{L} \widetilde{\nu}_{L}$ can dominate $\widetilde{l}_{R} \widetilde{l}_{R}$ even for $m_{\tilde{l}_{L}}, m_{\tilde{\nu}_{L}}>m_{\tilde{l}_{R}}$ because of the larger $\mathrm{SU}(2)_{L}$ gauge coupling for left-handed states. For $m_{\tilde{l}_{L}}, m_{\widetilde{\nu}_{L}}<m_{\chi_{2}^{0}}$ the lefthanded sleptons and sneutrinos decay predominantly by $\tilde{l}_{L} \rightarrow \chi_{1}^{0} l$ and $\tilde{\nu}_{L} \rightarrow \chi_{1}^{0} \nu$, leading to the final states $l^{+} l^{-} \gamma \gamma+\mathbb{E}_{T}, l^{ \pm} \gamma \gamma+\boldsymbol{E}_{T}$, and $\gamma \gamma+\boldsymbol{E}_{T}$ at comparable rates. In addition, for $m_{\tau_{L}}, m_{\widetilde{\nu}_{L}}<m_{\chi_{2}^{0}}, \chi_{2}^{0}$ and $\chi_{1}^{ \pm}$decay predominantly by $\chi_{2}^{0} \rightarrow \widetilde{l}_{L} l, \widetilde{\nu}_{L}, \nu$ and $\chi_{1}^{ \pm} \rightarrow \widetilde{l}_{L}^{ \pm} \nu, \widetilde{\nu}_{L} l^{ \pm}$. Pair production of $\chi_{1}^{+} \chi_{1}^{-}$then leads to the final states $l^{+} l^{\prime-} \gamma \gamma$

TABLE III. Production cross sections (fb) for each lepton flavor for $m_{\chi_{1}^{0}}=100 \mathrm{GeV}, \mu \gg m_{\chi_{1}^{0}}, m_{\tilde{l}_{R}}=110 \mathrm{GeV}, m_{\tilde{l}_{L}}=135 \mathrm{GeV}$, and $m_{\widetilde{\nu}_{L}}=120 \mathrm{GeV}$, as discussed in Sec. V. The center of mass energy is $1.8 \mathrm{TeV}$. Each final state has $\mathbb{E}_{T}$. The total cross sections in each channel are summed over all lepton flavors.

\begin{tabular}{ccccccc}
\hline \hline & $\gamma \gamma$ & $l^{ \pm} \gamma \gamma$ & $l^{+} l^{-} \gamma \gamma$ & $l^{+} l^{\prime-} \gamma \gamma$ & $l^{+} l^{-} l^{ \pm} \gamma \gamma$ & $l^{+} l^{-} l^{\prime \pm} \gamma \gamma$ \\
\hline$\widetilde{l_{L}} \widetilde{l}_{L}$ & - & - & 5.6 & - & - & - \\
$\widetilde{l_{L}} \widetilde{\nu}_{L}$ & - & 17.0 & - & - & - & - \\
$\widetilde{\nu_{L}} \widetilde{\nu}_{L}$ & 6.7 & - & - & - & - & - \\
$\widetilde{l}_{R} \widetilde{l}_{R}$ & - & - & 6.0 & - & - & - \\
$\chi_{2}^{0} \chi_{1}^{ \pm}$ & - & 6.5 & - & - & 1.65 & 1.65 \\
$\chi_{1}^{+} \chi_{1}^{-}$ & - & - & 2.1 & 2.1 & - & - \\
\hline Total & 20.0 & 70.5 & 41.1 & 12.6 & 5.0 & 9.9 \\
\hline \hline
\end{tabular}

$+\boldsymbol{E}_{T}$, and $\chi_{2}^{0} \chi_{1}^{ \pm}$leads to $l^{ \pm} \gamma \gamma+\boldsymbol{E}_{T}$ and $l^{+} l^{-} l^{\prime \pm}+\boldsymbol{E}_{T}$ final states. To illustrate this, the production cross sections for $m_{\tilde{l}_{L}}=135 \mathrm{GeV}, m_{\widetilde{\nu}_{L}}=120 \mathrm{GeV}$, and $m_{\tilde{l}_{R}}=110 \mathrm{GeV}$ are presented in Table III. The $\widetilde{l}_{L}-\widetilde{\nu}_{L}$ mass splitting is that which arises from the $\mathrm{SU}(2)_{L} \times \mathrm{U}(1)_{Y} \quad D$ terms, $m_{\widetilde{l}_{L}}^{2}-m_{\widetilde{\nu}_{L}}^{2}=-m_{W}^{2} \cos 2 \beta$, for $\tan \beta=2$. The lightest neutralinos are taken to be mostly gaugino, and the mass ratios of the right-handed sleptons to gauginos are taken to be those of the MGM model. It is interesting to note that with this spectrum, no jets result from the cascade decays.

Left-handed slepton pair production gives rise to very distinctive final states. In the $\sin ^{2} \theta_{W} \rightarrow 0$ and $\tan \beta \rightarrow 1$ limit, $\sigma\left(\widetilde{l_{L}}{\widetilde{\nu_{L}}}\right)=2 \sigma\left(\widetilde{l_{L}} \widetilde{l}_{L}\right)=2 \sigma\left(\widetilde{\nu}_{L} \widetilde{\nu}_{L}\right)$. Final states $l \gamma \gamma+\boldsymbol{E}_{T}$ and $\gamma \gamma+\boldsymbol{E}_{T}$ in roughly this ratio represent an important test for $\tilde{l}_{L} \widetilde{l}_{L}$ production. The relative rate of $l^{+} l^{-} \gamma \gamma+\boldsymbol{E}_{T}$ events depends on the mass of the right-handed slepton with respect to the left-handed slepton. A rate for $\widetilde{l}_{L} \widetilde{l}_{L}$ comparable to or larger than that for $\widetilde{l}_{R} \widetilde{l}_{R}$ would imply a mass spectrum which is not consistent with the MGM mass relations.

\section{IMPLICATIONS OF CURRENT DATA}

It is by now well known that a single event of the type $e^{+} e^{-} \gamma \gamma+\mathbb{E}_{T}$ has reportedly been observed by the CDF Collaboration [9]. The single event is consistent with $\widetilde{e}^{+} \widetilde{e}$ pair production, and subsequent decays $\widetilde{e} \rightarrow \chi_{1}^{0} e$ and $\chi_{1}^{0} \rightarrow \gamma G$, within low scale supersymmetry breaking $[6,8]$. For a single event in $\sim 100 \mathrm{pb}^{-1}$ of integrated luminosity, the $90 \%$ C.L. range for the cross section is roughly 5-40 fb. The kinematics of the event requires $m_{l} \gtrsim 60 \mathrm{GeV}$. If $\chi_{1}^{0}$ is mostly gaugino, the nonobservation of an excess in $e^{+} e^{-} \rightarrow \gamma \gamma+\boldsymbol{E}_{T}$ at the CERN $e^{+} e^{-}$collider LEP 135 [13] gives a bound on the $\chi_{1}^{0}$ mass almost to the kinematic threshold, $m_{\chi_{1}^{0}}>65 \mathrm{GeV}$. Using this, the kinematics of the $e^{+} e^{-} \gamma \gamma+\mathbb{E}_{T}$ event requires $m_{l} \gtrsim 90 \mathrm{GeV}$. If $e^{+} e^{-} \rightarrow \gamma \gamma+\boldsymbol{E}_{T}$ were not observed at LEP 190, this would increase to $m_{l} \gtrsim 110 \mathrm{GeV}$. Given the analysis of the previous sections, it is interesting to investigate what consequences the Goldstino interpretation of this event has for the messenger sector and for other channels which could be observed at the Tevatron and LEP II.

Within the MGM model, the most natural interpretation 


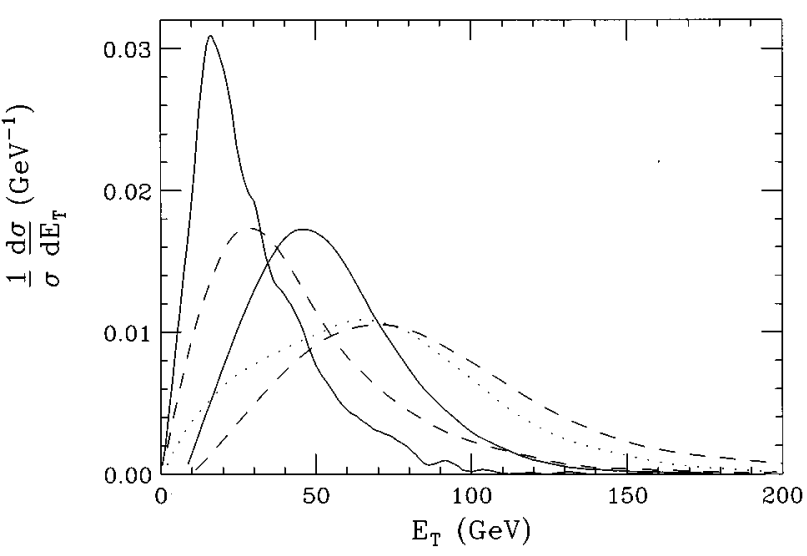

FIG. 2. The $E_{T}$ and $\mathbb{E}_{T}$ spectra for the $l^{+} l^{-} \gamma \gamma+\mathbb{E}_{T}$ final state with $m_{\tilde{l}}=135 \mathrm{GeV}$ and $m_{\chi_{1}^{0}}=100 \mathrm{GeV}$, as discussed in Sec. VI. The center of mass energy is $1.8 \mathrm{TeV}$. The two solid lines are the $E_{T}$ distributions of the hard and soft electrons. Similarly, the dashed lines are the $E_{T}$ distributions of the hard and soft photons. The dotted line is the $\mathbb{E}_{T}$ distribution.

would be $\widetilde{l}_{R} \widetilde{l}_{R}$ pair production. Based on the $\widetilde{l}_{R} \widetilde{l}_{R}$ cross section, this would imply a $90 \%$ C.L. range for $m_{\tilde{l}_{R}}$ of $70 \lesssim m_{\tilde{l}_{R}} \leqslant 115 \mathrm{GeV}$, consistent with the kinematic bounds given above. This interpretation is somewhat problematic for a number of reasons. All the partons in the event are fairly hard, $E_{T, e_{1}} \simeq 64 \mathrm{GeV}, E_{T, e_{2}} \simeq 34 \mathrm{GeV}, E_{T, \gamma_{1}} \simeq 32 \mathrm{GeV}$, and $E_{T, \gamma_{2}} \simeq 40 \mathrm{GeV}$. However, as discussed in Sec. II, within the MGM model the leptons in such events should be much softer on average than the photons. For the parameters given in Table I the probability that both leptons have $E_{T}>30 \mathrm{GeV}$ is $\lesssim 2 \%$. It is possible for the $m_{l_{R}}-m_{\chi_{1}^{0}}$ splitting to be larger than the MGM relation, as discussed in Sec. III, thereby increasing the average lepton $E_{T}$. Right-handed sleptons much heavier than $115 \mathrm{GeV}$ are, however, disfavored by the implied rate. Values of $m_{\chi_{1}^{0}}$ much smaller than $100 \mathrm{GeV}$ are disfavored if $\chi_{1}^{0}$ is mostly gaugino since $\chi_{2}^{0} \chi_{1}^{ \pm}$and $\chi_{1}^{+} \chi_{1}^{-}$ pair production would lead to an excessive rate for the final states $W W \gamma \gamma+\boldsymbol{E}_{T}$ and $W l^{+} l^{-}+\boldsymbol{E}_{T}$ or $W Z \gamma \gamma+\boldsymbol{E}_{T}$. We therefore conclude that the kinematics of the event is not easily accommodated by $\widetilde{l_{R}} \widetilde{l}_{R}$ production if $\chi_{1}^{0}$ is an electroweak neutralino.

It is worth noting in passing that it is possible for neutralino pair production to result in $l^{+} l^{-} \gamma \gamma+\boldsymbol{E}_{T}$ over some range of parameters, as discussed in Sec. IV. However, the kinematics and many other concomitant final states also disfavor this interpretation.

The problem of obtaining hard leptons is largely ameliorated if the event is interpreted as arising from $\widetilde{l}_{L} \widetilde{l}_{L}$ pair production. The larger gauge coupling of the left-handed sleptons relative to the right-handed ones results in a larger intrinsic cross section. Based on the $\widetilde{l}_{L} \widetilde{l}_{L}$ cross section, the $90 \%$ C.L. range for $m_{\tilde{l}_{L}}$ is $85 \lesssim m_{\tilde{l}_{L}} \lesssim 135 \mathrm{GeV}$. This allows for a larger $m_{\tau_{L}}-m_{\chi_{1}^{0}}$ splitting, resulting in harder leptons. As an example, the $E_{T}$ and $\boldsymbol{E}_{T}$ spectra for $m_{\chi_{1}^{0}}=100 \mathrm{GeV}$ and $m_{\tilde{l}_{L}}=135 \mathrm{GeV}$ are shown in Fig. 2. For these parameters the photon and lepton averages $E_{T}$ are of the same order.
This interpretation has a number of interesting consequences. First, as discussed in Sec. V, left-handed slepton and sneutrino production gives rise to the additional final states $l^{ \pm} \gamma \gamma+\mathbb{E}_{T}$ and $\gamma \gamma+\mathbb{E}_{T}$. The rate for these final states should be roughly in the ratio $2: 1$ (depending precisely on the value of $\tan \beta$ ) and comparable to the $l^{+} l^{-} \gamma \gamma+\boldsymbol{E}_{T}$ rate. In addition, if $\chi_{1}^{0}$ is mostly gaugino and $m_{\tilde{l}_{L}}, m_{\widetilde{\nu}_{L}}<m_{\chi_{2}^{0}}$, $\chi_{2}^{0} \chi_{1}^{ \pm}$and $\chi_{1}^{+} \chi_{1}^{-}$production should give rise to final states $X \gamma \gamma+\boldsymbol{E}_{T}$, where $X=l^{ \pm}, l^{+} l^{\prime-}, l^{+} l^{-} l^{\prime \pm}$ at slightly reduced rates. However, if $\chi_{1}^{0}$ were mostly electroweak singlet, the rate for these final states would be suppressed.

\section{CONCLUSIONS}

If supersymmetry is broken at a low scale, the lightest standard model supersymmetric particle can decay to its partner plus the Goldstino. This leads to the possibility of signatures which are quite distinct from those of high scale supersymmetry breaking [6]. Here we investigated the phenomenology at the Tevatron for the case in which the lightest standard model superpartner is a neutralino. The generic feature for this case is a final state with two hard photons and missing transverse energy. The additional partons in the final state are sensitive to the superpartner mass spectrum and can depend indirectly on details of the messenger sector. An observation of $l^{+} l^{-} \gamma \gamma+\boldsymbol{E}_{T}$ alone with soft leptons and hard photons would be a good indication of right-handed slepton pair production. The final states $W W \gamma \gamma+\mathbb{E}_{T}$ and the sum of $W l^{+} l^{-} \gamma \gamma+\boldsymbol{E}_{T}$ and $W Z \gamma \gamma+\boldsymbol{E}_{T}$ in approximately a 2:1 ratio result if the lightest two neutralinos and lightest chargino are mostly gaugino and $m_{\tilde{l}_{L}}, m_{\widetilde{v}_{L}}>m_{\chi_{2}^{0}}$. The combination of the additional final states $j j \gamma \gamma+\boldsymbol{E}_{T}$ and $l^{ \pm} \gamma \gamma+\boldsymbol{E}_{T}$ can arise if the lightest neutralino has a significant Higgsino component. Finally, the final states $l^{ \pm} \gamma \gamma+\boldsymbol{E}_{T}, l^{+} l^{-} \gamma \gamma+\boldsymbol{E}_{T}$, and $\gamma \gamma+\boldsymbol{E}_{T}$ arise in approximately a 2:1:1 ratio from left-handed slepton production. If $m_{\tilde{l}_{L}}, m_{\widetilde{\nu}_{L}}<m_{\chi_{2}^{0}}$ the additional final states $l^{+} l^{\prime-} \gamma \gamma+\boldsymbol{E}_{T}$ and $l^{+} l^{-} l^{\prime \pm} \gamma \gamma+\mathbb{E}_{T}$ are also significant. If the ordinary gauge interactions are the messengers for supersymmetry breaking, all final states discussed in this paper will occur with equal rates for each generation. Violations of lepton universality in two-photon events would likely indicate a much richer family-dependent messenger sector.

The kinematics of the above final states is sensitive to the mass spectrum. Since the photons arise at the end of the decay chain, their $E_{T}$ spectrum is generally flatter than for the other partons. For very massive superpartners the splitting between states is typically smaller than the overall scale, giving rise to an average photon $E_{T}$ much larger than for the other partons, which originate further up the decay chain. For superpartners which could be observed with the current integrated luminosity at the Tevatron, this is, however, not necessarily the case (cf. Fig. 2).

With current or anticipated integrated luminosities, the signatures $X \gamma \gamma+\boldsymbol{E}_{T}$ discussed in this paper have very small standard model backgrounds. The largest potential backgrounds are probably misidentifications. The most problematic of these could be hard $\pi^{0} \pi^{0}$ pairs which are interpreted as $\gamma \gamma$. However, in the final states with leptonic activity only, this requires that two hard jets each fluctuate to a single $\pi^{0}$ plus hadronic activity below the pedestal. This doubly 
rare fluctuation could be estimated if the single rare fluctuation rate can be characterized experimentally.

Given these very distinctive final states with negligible standard model backgrounds, it is interesting to ask what the discovery reach will be at future hadron colliders. For a fiveevent signal at the Tevatron with $\sqrt{s}=2 \mathrm{TeV}$, pair production of $\widetilde{l_{R}} \widetilde{l}_{R}$ and $\chi_{2}^{0} \chi_{1}^{ \pm}$gives mass reaches of $m_{\tilde{l}_{R}} \leqslant 145$ (220) $\mathrm{GeV}$ and $m_{\chi_{1}^{ \pm}} \lesssim 300$ (380) GeV for an integrated luminosity of $2(20) \mathrm{fb}^{-1}$. Notice that these are well beyond the reach of LEPII. Since the right-handed sleptons are generally lighter than the chargino, both processes probe roughly the same overall scale. The analogous processes at the CERN Large Hadron Collider (LHC) with $\sqrt{s}=14 \mathrm{TeV}$ give mass reaches $m_{\widetilde{l}_{R}} \lesssim 540 \mathrm{GeV}$ and $m_{\chi_{1}^{ \pm}} \lesssim 1200 \mathrm{GeV}$ for an integrated luminosity of $30 \mathrm{fb}^{-1}$. In contrast, the reach for the oftendiscussed case of high scale supersymmetry breaking is significantly lower because of standard model backgrounds $[14,15]$.

Throughout, we have assumed that $\chi_{1}^{0}$ decays promptly by $\chi_{1}^{0} \rightarrow \gamma G$. However, for a supersymmetry-breaking scale of a few thousand $\mathrm{TeV}$ this decay length can be on the same scale as the detector dimensions [6]. With some fraction of the decays taking place outside the detector, some events could appear with a single photon or without photons. In addition, if $\chi_{1}^{0}$ has a non-negligible Higgsino component, the decay $\chi_{1}^{0} \rightarrow h^{0} G$ can arise, where $h^{0}$ is the lightest Higgs boson. Some fraction of the events would then have one or both photons replaced by $b b$ jets reconstructing the Higgs boson mass. This would represent a very interesting, and relatively clean, source for Higgs bosons.
The single $e^{+} e^{-} \gamma \gamma+\mathbb{E}_{T}$ event observed at the Tevatron by the CDF Collaboration [9] is most naturally interpreted as low scale supersymmetry breaking, with the missing energy carried by Goldstinos. The relatively hard leptons and softer photons, and lack of many other events in other channels, suggest $\widetilde{l}_{L} \widetilde{l}_{L}$ pair production as the origin of this event. In this promising scenario $\widetilde{l_{L}} \widetilde{\nu_{L}}$ and $\widetilde{\nu_{L}} \widetilde{\nu_{L}}$ pair production gives rise to $l^{ \pm} \gamma \gamma+\mathbb{E}_{T}$ and $\gamma \gamma+\mathbb{E}_{T}$ final states at comparable rates. If these events are not seen after a complete analysis of the current $\mathrm{CDF}$ and D0 data, this interpretation would be somewhat problematic. In addition, for $m_{\tau_{L}}, m_{\widetilde{\nu}_{L}}>m_{\chi_{2}^{0}}$, the final states $W W \gamma \gamma+\mathbb{E}_{T}$ and either $W Z \gamma \gamma+\boldsymbol{E}_{T}$ or $W l^{+} l^{-} \gamma \gamma+\mathbb{E}_{T}$ can test the gaugino fraction of $\chi_{1}^{0}$. Alternately, if $m_{\tilde{l}_{L}}, m_{\widetilde{\nu}_{L}}<m_{\chi_{2}^{0}}$ the final states $l^{+} l^{\prime-} \gamma \gamma+\boldsymbol{E}_{T}$ and $l^{+} l^{-} l^{\prime \pm} \gamma \gamma+\boldsymbol{E}_{T}$ can test the gaugino fraction of $\chi_{1}^{0}$. If $\chi_{1}^{0}$ is mostly gaugino, these are likely to be seen in the current data. In contrast, if $\chi_{1}^{0}$ is mostly singlet, the rate for these final states would be reduced.

Finally, it is worth commenting on the implications of this interpretation of the CDF event for LEPII. Our analysis indicates that slepton pair production is likely to be out of reach at LEPII. However, neutralino pair production is not necessarily out of reach. Its signature would be spectacular $\gamma \gamma+\boldsymbol{E}_{T}$ events with a coplanar photons.

\section{ACKNOWLEDGMENTS}

We would like to thank M. Dine and H. Haber for useful discussions. The work of S.T. and J.D.W. was supported by the Department of Energy under Contract No. DE-AC0376 SF00515.
[1] M. Dine, W. Fischler, and M. Srednicki, Nucl. Phys. B189, 575 (1981); S. Dimopoulos and S. Raby, ibid. B192, 353 (1981); M. Dine and W. Fischler, Phys. Lett. 110B, 227 (1982); M. Dine and M. Srednicki, Nucl. Phys. B202, 238 (1982); L. Alvarez-Gaumé, M. Claudson, and M. Wise, ibid. B207, 96 (1982); C. Nappi and B. Ovrut, Phys. Lett. 113B, 175 (1982).

[2] M. Dine and W. Fischler, Nucl. Phys. B204, 346 (1982); S. Dimopoulos and S. Raby, ibid. B219, 479 (1983).

[3] P. Fayet, Phys. Lett. 70B, 461 (1977); 84B, 416 (1979); 84B, 241 (1979).

[4] M. Dine, A. E. Nelson, and Y. Shirman, Phys. Rev. D 51, 1362 (1995).

[5] S. Dimopoulos, M. Dine, and S. Thomas, presented at ITP Workshop on SUSY Phenomena and SUSY GUTs, Santa Barbara, CA, 1995 (unpublished).

[6] S. Dimopoulos, M. Dine, S. Raby, and S. Thomas, Phys. Rev. Lett. 76, 3494 (1996).

[7] For the latest analysis, see S. Ambrosanio and B. Mele, Phys. Rev. D 53, 2541 (1996).

[8] S. Ambrosanio, G. L. Kane, G. Kribs, S. P. Martin, and S. Mrenna, Phys. Rev. Lett. 76, 3498 (1996).

[9] CDF Collaboration, S. Park, in 10th Topical Workshop on
Proton-Antiproton Collider Physics, edited by R. Raha and J. Yoh (AIP Press, New York, 1995).

[10] G. Dvali, G. Giudice, and A. Pomarol, Report No. CERN-TH96-61, hep-ph/9603238 (unpublished).

[11] M. Dine, A. E. Nelson, Y. Nir, and Y. Shirman, Phys. Rev. D 53, 2658 (1996).

[12] We use ISAJET and ISASUSY for event generation: T. Sjöstrand, Comput. Phys. Commun. 46, 43 (1987); H. Baer, F. Paige, S. Protopescu, and X. Tata, in Proceedings of the Workshop on Physics at Current Accelerators and the Supercolliders, Argonne, Illinois, 1993, edited by J. L. Hewett et al. (Argonne Report No. ANL-HEP-CP-93-92, Argonne, 1993), Report No. hep-ph/9305342 (unpublished).

[13] The OPAL Collaboration, G. Alexander et al., Report No. CERN-PPE/96-039 (unpublished).

[14] H. Baer, K. Hagiwara, and X. Tata, Phys. Rev. D 35, 1598 (1987); P. Nath and R. Arnowitt, Mod. Phys. Lett. A 2, 331 (1987).

[15] H. Baer, C.-H. Chen, F. Paige, and X. Tata, Phys. Rev. D 49, 3238 (1994); 50, 450 (1994); H. Baer, C.-H. Chen, C. Kao, and X. Tata, ibid. 52, 1565 (1995); S. Mrenna, G. Kane, G. Kribs, and J. D. Wells, ibid. 53, 1168 (1996). 